AN ANALYSIS OF AN BTECTROMAGNETICATLY DRIVEN CONFINED VORTEXX

WITH HIGH POSITIVE RADIAL REYNOIDS NUMBERS

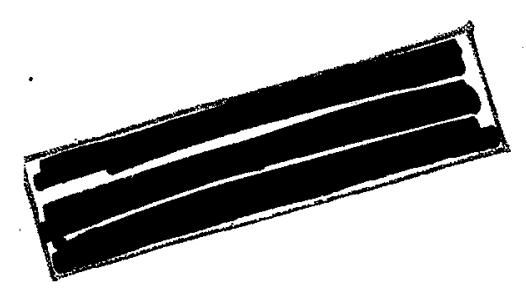

Roger B. Stewart

Presented at the AIAA Joint Electric Propulsion

and Plasmadynamics Conference

NASA Langley Research Center

Iangley Station, Hampton, Va.
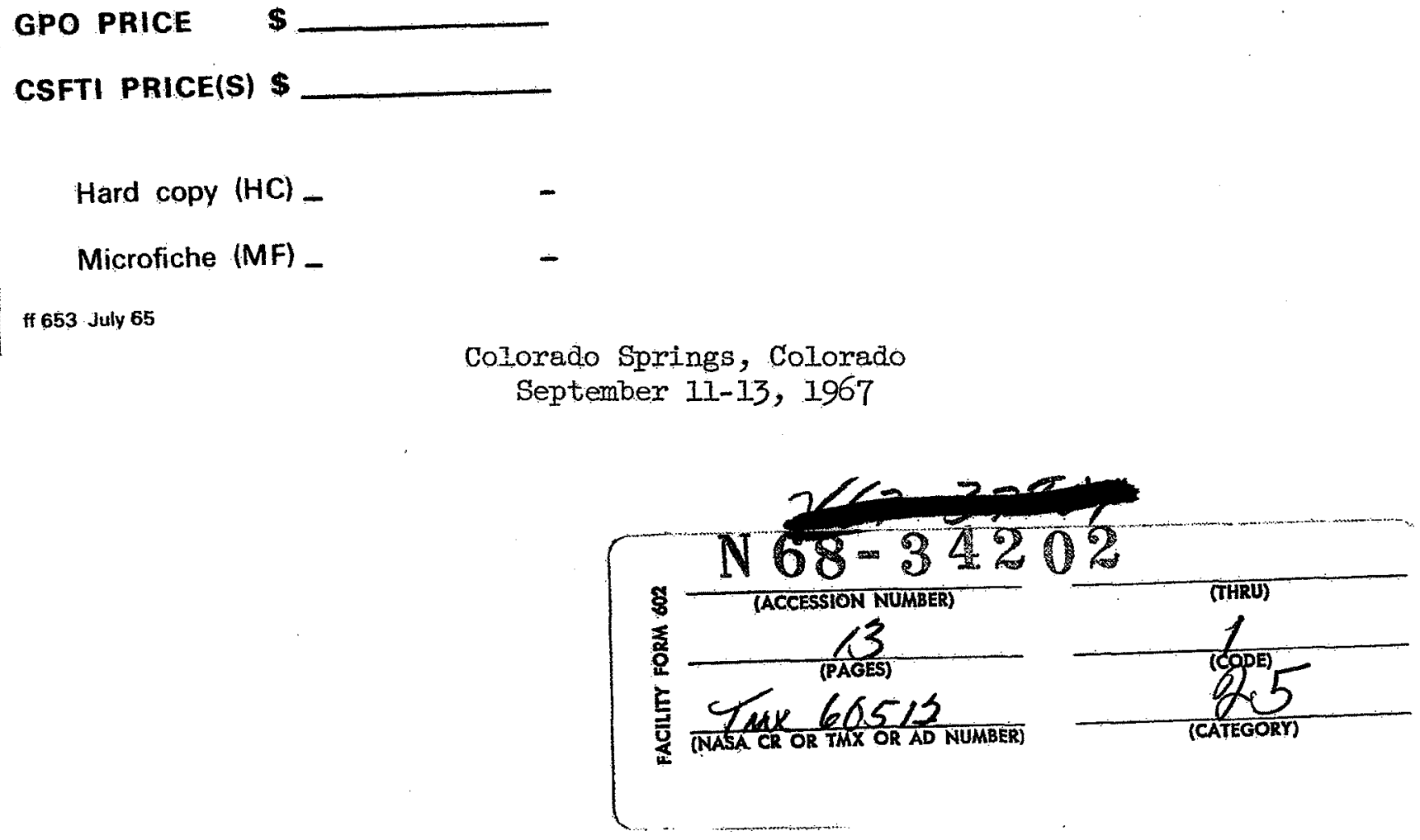


\title{
AN ANALYSIS OF AN BLECTROMAGNEITCALLY DRIVEN CONFINED VORTEX WITH HIGH POSITIVE RADIAI REYNOLDS NUMBERS
}

\author{
Roger B. Stewart \\ Aerospace Engineer \\ NASA Langley Research Center \\ Langley Station, Hampton, Va.
}

\section{Abstract}

The flow behavior in an electromagnetically driven viscous vortex is investigated for a concentric, cylindrical electrode geometry with outward radial mass flow through the chamber. The closedform solutions obtained by Kessey and also Lewellen for the velocity, pressure, and induced magnetic field distributions with inward radial flow are applied to the present study with the assumption of small magnetic Reynolds numbers and negligible flow variations in the axial direction. An approximate form of the energy equation that includes a temperature dependent electrical conductivity is solved numerically for the temperature distribution across the vortex. For the case of constant radial current flow, the applied voltage and input power are also calculated. The results of this study are compared with experimental measurements made with several arc-driven vortex accelerator configurations. The theoretical and experimental results are in agreement with regard to the velocities in the outer portion of the vortex, the inner and outer boundary pressures, and the power required to heat and accelerate the flow. Significantly, the solid-body-like rotation predicted by this analysis appears to be present in the experimental devices.

\section{Introduction}

te present study deals with the flow behavior xisymmetric, electromagnetically driven, ; vortex that is confined between cylindrical electrodes, and has a net outward radial mass flow. The widespread interest in the production of continuous hypervelocity nozzle flows provided the motivation for this analysis and for the experiments with several vortex accelerator configurations that are reported here.

A number of analytical studies have been reported in recent years in which a plasma moves within a cylindrical geometry. McCune ${ }^{(1)}$ and Donaldson(2) obtained solutions for the flow variables in a coaxial cylindrical system neglecting

induced magnetic field effects. Lewellen (3) obtained analytic solutions for the velocity, pressure, temperature, and induced magnetic field distributions in a "long cylinder" electrode geometry for the case of small magnetic Reynolds numbers.

Kessey (4) analyzed both the inviscid zero mass flow and viscous nonzero mass flow problem and obtained solutions for the velocity, pressure, and induced magnetic field distributions within a coaxial cylindrical geometry. All of these studies have considered either the case of deceleration due to electromagnetic interaction, or acceleration of the plasma with zero mass flow, or a net inward radial mass

flow. Although the mathematical nature of the solum tions to the equations that govern the vortex is the same regardless of whether the flow is radially inward or outward, the flow within the vortex is drastically altered from the inflow case if the mass flow is in the outward direction. This is true because the azimuthal velocity has an exponential dependence on the radius. The exponent is the radial Reynolds number which is positive for outflow but negative for inflow.

The practical significance of an electromagnetically driven vortex with outward radial mass flow has been demonstrated by Stewart and Wallio (5) and the theoretical and experimental results of the present work provide some insight into the nature of the high energy gas flow that exists within these devices.

\section{Symbols}

$\widetilde{\mathbb{B}}$

$\mathrm{E}$

$G_{1}$

$h_{\mathrm{O}}$

ht

I

$J$

$\mathrm{k}$

$p$

$r$

V

$\tilde{\mathrm{V}}$

$\rho$ plasma density

$\eta \quad$ viscosity

$\theta$ azimuthal coordinate

$\Phi \quad$ applied voltage between the electrodes

$\mu \quad$ magnetic permeability

Subscripts: o electrical conductivity 
1 quantities evaluated at the inner wall

$r, \theta, z \quad$ quantity along a coordinate axis

Principal dimensionless quantities:

$\zeta=\frac{r}{r_{0}} \quad$ radial coordinate

$\xi=\frac{z}{r_{0}} \quad$ axial coordinate

$\mathrm{v}=\frac{\mathrm{V}_{\theta}}{\tilde{\mathrm{V}}} \quad$ azimuthal velocity

$\mathrm{b}=\frac{\mathrm{B}_{\theta}}{\widetilde{B}} \quad$ azimuthal magnetic flux

$P=\frac{p}{\widetilde{B}^{2} / \mu} \quad$ pressure

$\mathrm{R}_{\mathrm{er}}=\frac{\rho \mathrm{V}_{\mathrm{r}} \mathrm{r}}{\eta} \quad$ radial Reynolds number

$M=\frac{V_{\theta}}{\sqrt{\gamma \mathrm{RTZ}^{2}}} \quad$ Mach number

$\mathrm{H}_{\mathrm{a}}=\mathrm{B}_{\mathrm{applied}} \mathrm{r}_{\mathrm{\sigma}} \sqrt{\eta} \quad$ Hartmann number

$\mathrm{R}_{\mathrm{em}}=\mu \sigma \mathrm{V}_{\mathrm{r}} \mathrm{r} \quad$ radial magnetic Reynolds number

Rationalized MKS units are used.

Basic Considerations

The flow system under consideration consists of an axisymmetric electrically conaucting viscous vortex confined in a long annular region such that axial flow variations due to end-wall effects are negligible. The electrode surfaces are perfectly conducting and porous to allow for mass flow through the vortex. Fluid motion exists in the azimuthal and outward radial directions and figure 1 shows the electrode geometry and basic nomenclature. The conventional magnetohydrodynamic equations ${ }^{(6)}$ are used with a right-hand cylindrical coordinate sys-

tem. The following principal assumptions are made:

1. The radial magnetic Reynolds number is small.

2. The plasma is incompressible.

3. The axial velocity is negligible as well as axial variations of the azimuthal velocity.

4. The vortex is axisymmetric.

These are essentially the same assumptions dis discussed in detail in references 1 to 4 . By virtue of assumption 1 , the Hall current can be neglected. With constant density from assumption 2 and using assumptions 3 and 4, closed-form solu-

tions can be obtained for the radial momentum equation and the equation determining the induced azimuthal magnetic field. For nonzero radial mass flow the azimuthal momentum equation does not require a constant density assumption and is valid for both compressible and incompressible flow if the radial Reynolds number is constant. Assumption 3 is the long cylinder approximation that amounts to neglecting the effects produced by end walls in a finite length chamber. Assumption 4 provides a convenient simplification to the governing equations.

With these assumptions it has been shown $(7)$

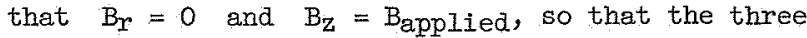
components of the momentum equation and the equation for the induced azimuthal magnetic field can be written as shown in the following section.

Governing Equations

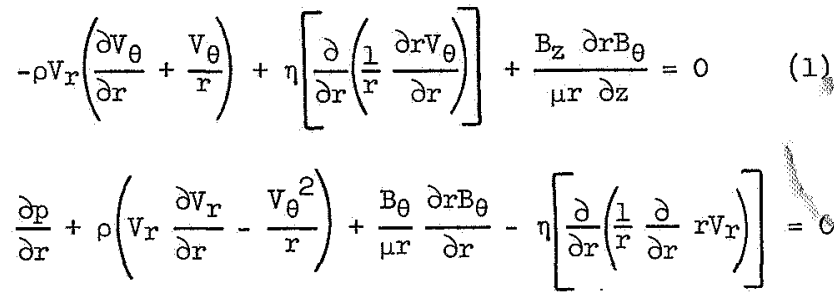

$$
\frac{\partial p}{\partial z}+\frac{B_{\theta}}{\mu} \frac{\partial B_{\theta}}{\partial z}=0
$$

$\frac{1}{\mu \sigma}\left[\frac{\partial}{\partial r}\left(\frac{1}{r} \frac{\partial r B_{\theta}}{\partial r}\right)+\frac{\partial^{2} B_{\theta}}{\partial z^{2}}\right]-V_{r}\left(\frac{\partial B_{\theta}}{\partial r}-\frac{B_{\theta}}{r}\right)=0$

Several simplifications can be obtained for this system. Integrating the continuity equation gives

$$
r V_{r}=C_{1}
$$

and from the radial component of Ampere's law when $I_{r}=$ constant

$$
-\frac{1}{\mu r} \frac{\partial r B_{\theta}}{\partial z}=J_{r}
$$

and

$$
\frac{\partial^{2} B_{\theta}}{\partial z^{2}}=0
$$

Thus, equations (1) and (4) are two second order differential equations for $V_{\theta}$ and $B_{\theta}$.

The boundary conditions for $V_{\theta}$ and $B_{\theta}$ are the following:

$$
\begin{array}{lll}
\mathrm{V}_{\theta}=\mathrm{V}_{\theta_{1}} & \text { at } & r=r_{1} \\
\mathrm{~V}_{\theta}=0 & \text { at } & r=r_{0}
\end{array}
$$

Where $V_{\theta_{1}}$ results from either a rotating inner electrode or azimuthal gas injection through the 
electrode surface. Using the $\mathrm{z}$ component of $\mathrm{Ohm}$ 's law and Ampere's law, two boundary conditions for $\mathrm{B}_{\theta}$ can be determined, thus:

$$
\begin{array}{lll}
\frac{\partial\left(\mathrm{rB}_{\theta}\right)}{\partial x}=R_{\mathrm{em}} \mathrm{B}_{\theta} & \text { at } & r=r_{1} \\
& \text { at } & r=r_{0}
\end{array}
$$

The external circuit chosen for this study provides a symmetrical current flow through each end of the center electrode. Such a circuit configuration is needed in order to avoid the net axial

Lorentz force on the plasma that would be present if all the current were introduced through only one end of the center electrode. Thus, the end wall boundary conditions on the induced azimuthal magnetic field are:

$$
\begin{array}{lll}
B_{\theta}=\frac{-I_{r}}{2}\left(\frac{\mu}{2 \pi r}\right) & \text { at } & z=h_{0} \\
B_{\theta}=\frac{I_{r}}{2}\left(\frac{\mu}{2 \pi r}\right) & \text { at } & z=-h_{O}
\end{array}
$$

In order to work with dimensionless equations, the dimensional reference quantities $\widetilde{V}$ and $\widetilde{B}$ for the velocity and magnetic induction will be defined by the expression: $\widetilde{B}=\widetilde{V} \sqrt{\rho \mu}$ where $\rho$ is the plasma density. The following dimensionless quantities are thus defined:

$$
\begin{aligned}
& \zeta=\frac{r}{r_{0}} \quad \xi=\frac{z}{r_{0}} \quad b=\frac{B_{\theta}}{\widetilde{B}} \quad v=\frac{V_{\theta}}{\widetilde{V}} \quad u=\frac{V_{r}}{\widetilde{V}} \\
& P=\frac{p}{\frac{P}{2}} \quad N=\frac{B_{\text {applied }}}{\widetilde{B}} \quad i=\frac{I_{r}}{2 \pi h_{0} \frac{\widetilde{B}}{\mu}} \quad P_{w r}=\frac{\text { Power }}{\frac{\widetilde{B}}{\mu} \tilde{V} r_{O} h_{0}}
\end{aligned}
$$

and

$\varphi=\frac{\Phi}{\widetilde{B} r_{r_{0}}} \quad$ the dimensionless applied voltage $\lambda_{1}=\frac{G_{1}}{\rho \tilde{V}} \quad \begin{aligned} & \text { the dimensionless radial mass flow } \\ & \text { where } G_{1}=\rho_{1} V_{r_{1}} \text { the radial mass }\end{aligned}$ flow rate per unit area at the inner electrode surface

$\frac{1}{\lambda_{2}}=\frac{\rho r_{0} \tilde{V}}{\eta} \quad$ reference radial Reynolds number $\frac{1}{\lambda_{3}}=\mu \sigma r_{0} \tilde{V} \quad \begin{gathered}\text { reference radial magnetic Reynolds } \\ \text { number }\end{gathered}$

$\widetilde{\mathrm{B}}$ has been taken as 1.0 weber/meter ${ }^{2}$ for all of the calculations presented. With these definitions equations ( 1 ) to (4) with the boundary conditions given by equations (8) to (13) can be written in dimensionless form as follows:

$$
\frac{d^{2} v}{d \zeta^{2}}+\frac{1}{\zeta}\left(1-R_{e r}\right) \frac{d v}{d \zeta}-\frac{v}{\zeta^{2}}\left(1+R_{e r}\right)=\frac{N i r_{0}}{2 \lambda_{2} h_{0} \zeta}
$$

$$
\frac{\partial P}{\partial \zeta}+b \frac{\partial b}{\partial \zeta}=\frac{\lambda_{1}^{2} \zeta_{1}^{2}}{\zeta^{3}}+\frac{1}{\zeta}\left(v^{2}-b^{2}\right)
$$

$$
\frac{\partial P}{\partial \xi}=-\frac{\partial \frac{b^{2}}{2}}{\partial \xi}
$$

$$
\frac{\partial^{2} \mathrm{~b}}{\partial \zeta^{2}}+\frac{1}{\zeta} \frac{\partial \mathrm{b}}{\partial \zeta}\left(1-\mathrm{R}_{\mathrm{em}}\right)+\frac{\mathrm{b}}{\zeta^{2}}\left(\mathrm{R}_{\mathrm{em}}-1\right)=0
$$

The dimensionless equations (14) and (17) have the boundary conditions.

$$
\begin{array}{lll}
\mathrm{v}=\mathrm{v}_{1} & \text { at } & \zeta=\zeta_{1} \\
\mathrm{v}=0 & \text { at } & \zeta=1
\end{array}
$$

$b=-\frac{i}{2 \zeta} \frac{h_{0}}{r_{0}} \quad$ at $\quad \xi=\frac{h_{0}}{r_{0}}$

$b=\frac{i}{2 \zeta} \frac{h_{0}}{r_{0}} \quad$ at $\quad \xi=-\frac{h_{0}}{r_{0}}$

$$
\frac{\partial \zeta \mathrm{b}}{\partial \zeta}=R_{\mathrm{em}} \mathrm{b} \doteq 0 \quad \text { at } \quad \zeta=\zeta_{I}
$$

Solutions

The solutions to equations (14) and (17) that satisfy the boundary conditions ( 18 to 23) ean be obtained in a straightforward manner similar to that worked out by Kessey. (4) Thus, equation (14) has the general solution for nonzero radial mass flow

$$
-\mathrm{v}=-\mathrm{C}_{2} \zeta^{\mathrm{I}+\mathrm{R}_{\mathrm{er}}}-\frac{\mathrm{C}_{3}}{\zeta}+\frac{\mathrm{Ni} \zeta}{4 \lambda_{1} \zeta_{1}}
$$

where

$$
C_{2}=\frac{N i}{4 \lambda_{1} \zeta_{1}}-\left[\frac{v_{1}+\frac{N i}{4 \lambda_{1}}\left(1-\zeta_{1}^{R_{e r}}\right)}{\frac{1}{\zeta_{1}}-\zeta_{1}^{1+R_{e r}}}\right]
$$

and

$$
C_{3}=-C_{2}+\frac{N i}{4 \lambda_{1} \zeta_{1}}
$$


For an applied magnetic field and a radial current in the positive $z$ and $r$ directions, respectively, the azimuthal velocity is in the negative $\theta$ direction (hence the negative sign on $v$ in eq. (24)).

A solution to equation (17) that satisfies equations (22) and (23) is

$$
\mathrm{b}=\left(\mathrm{a}_{1}+\mathrm{a}_{2} \xi\right) \frac{1}{\xi}
$$

and substituting the boundary conditions of equations (20) and (21) into equation (25) gives

$-\frac{i}{2 \zeta} \frac{h_{0}}{r_{0}}=\frac{a_{1}}{\zeta}+\frac{a_{2}}{\zeta}\left(\frac{h_{0}}{r_{0}}\right)$ at $\quad \xi=\frac{h_{0}}{r_{0}}$

and

$\frac{i}{2 \zeta} \frac{h_{0}}{r_{0}}=\frac{a_{1}}{\zeta}+\frac{a_{2}}{\zeta}\left(-\frac{h_{0}}{r_{0}}\right)$ at $\xi=-\frac{h_{0}}{r_{0}}$

From equations (26), $a_{1}$ is found to be zero so that the solution to equation (17) that satisfies the boundary conditions in equations (20) to (23) is

$$
\mathrm{b}=-\frac{1}{2 \zeta} \xi
$$

With $v$ and $b$ given by equations (24) and (27) the pressure distribution can be obtained by first integrating equation (16) to get

$$
P=-\frac{b^{2}}{2}+f_{1}(\zeta)
$$

and so

$$
\frac{\partial P}{\partial \zeta}=-\frac{\partial \frac{b^{2}}{2}}{\partial \zeta}+\frac{d f_{1}(\zeta)}{d \zeta}
$$

From equations (15) and (29) it is seen that:

$$
\frac{\partial f_{1}(\zeta)}{d \zeta}=\frac{\partial \frac{b^{2}}{2}}{\partial \zeta}-\frac{\partial \frac{b^{2}}{2}}{\partial \zeta}+\frac{\lambda_{1}^{2} \xi_{1}^{2}}{\zeta^{3}}+\frac{I}{\zeta}\left(v^{2}-b^{2}\right)
$$

Equation (30) can be integrated to give:

$$
f_{1}(\zeta)=-\frac{\lambda_{1}^{2} \zeta_{1}^{2}}{2 \zeta^{2}}+\int\left(v^{2}-b^{2}\right) \frac{d \zeta}{\zeta}+c_{4}
$$

Substituting equation (31) into equation (28) and evaluating the integral for $-b^{2} / \zeta$ yields

$$
P=-\frac{\lambda_{1}^{2} \zeta_{1}^{2}}{2 \zeta^{2}}+\int v^{2} \frac{d \zeta}{\zeta}+C_{4}
$$

The pure constant of integration $\mathrm{C}_{4}$ is eliminated by evaluating equation (32) at one boundary and subtracting the resultant expression from equation (32). In this study the pressure at the outer boundary $p_{0}$ will be chosen to eliminate $C_{4}$. Thus the pressure distribution for the case of net outward radial mass flow is found.

The applied voltage is given by

$$
\Phi=-\int_{\zeta_{I}}^{I} E_{r} r_{0} d \zeta
$$

which can be written in dimensionless form as

$$
\varphi=-\int_{\zeta_{1}}^{1} \frac{i \lambda_{3}}{2} \frac{d \zeta}{\zeta}+\int_{\zeta_{1}}^{1} \mathrm{Nv} d \zeta
$$

For the case of constant electrical conductivity, equation (34) can be integrated directly. For a temperature dependent electrical conductivity, $\lambda_{3}$. in equation (34) is replaced by its definition in terms of $\sigma$ and a numerical integration can be carried out once $\sigma(\zeta)$ is found from the temperature distribution across the flow.

The power input required to heat and accelerate the flow is given by

$$
\text { Power }=\int_{\zeta_{1}}^{1}\left(\frac{J_{r}^{2}}{\sigma}-v_{\theta} B_{z} J_{r}\right) 4 \pi h_{0} r_{0}^{2} \zeta d \zeta
$$

and in dimensionless form equation (35) is

$$
\mathrm{P}_{\mathrm{wS}}=\pi \mathrm{i} \int_{\zeta_{1}}^{1}\left(\frac{i \lambda_{3}}{\zeta}-2 \mathrm{vN}\right) d \zeta
$$

For a variable electrical conductivity equation (36) is numerically integrated in a manner similar to equation (34).

A determination of the temperature distribution can be obtained from the energy equation using the long cylinder approximation considered in this study. Because the joule heating terms can be expected to have a predominant effect on the temperature distribution, a useful approach is that of assuming that the viscosity and thermal conductivity are constant and that $\sigma$ varies in a known manner with temperature. With these simplifying assumptions the energy equation can be written as

$\rho \mathrm{C}_{\mathrm{v}} \mathrm{V}_{r} \frac{\mathrm{dT}}{\mathrm{dr}}=\frac{\mathrm{J}_{r}^{2}}{\sigma}+\eta\left(\frac{d \mathrm{~V}_{\theta}}{\mathrm{dr}}-\frac{\mathrm{V}_{\theta}}{\mathrm{r}}\right)^{2}+\frac{\mathrm{k}}{\mathrm{r}} \frac{\mathrm{d}}{\mathrm{dr}}\left(\mathrm{r} \frac{\mathrm{dT}}{\mathrm{dr}}\right)$

Rewriting equation (37) in terms of the dimensionless radial coordinate $\zeta$ and rearranging gives

$$
\begin{aligned}
\frac{d^{2} T}{d \zeta^{2}} & +\frac{1}{\zeta}\left(I-R_{e r} P_{r}+\rho \frac{r_{o} \zeta V_{r}}{k} R\right) \frac{d T}{d \zeta}+\frac{\eta}{k}\left(\frac{d V_{\theta}}{d \zeta}-\frac{V_{\theta}}{\zeta}\right)^{2} \\
& +\frac{J_{r}^{2} r_{o}^{2}}{\sigma k}=0
\end{aligned}
$$


where $P r$ is the PrandtI number, $R$ is the gas constant and $\mathrm{C}_{\mathrm{P}}-\mathrm{C}_{\mathrm{V}}=\mathrm{R}$ has been used. Equa-

tion (38) was numerically integrated using a temperature dependent electrical conductivity that was obtained from the data of reference 7 . A fifth degree polynomial was used to curve fit the electrical conductivity of equilibxium air at a pressure of 1 atmosphere. The integration was carried out on a computing machine using a classical fourth order Runge-Kutta. formula.

\section{Theoretical Results}

The solutions of the previous equations for the behavior of the vortex exhibit many interesting and widely different features from previously studied electromagnetically driven vortex flows. The most pronounced effects are due to the high positive radial Reynolds numbers (corresponding to outward radial mass flow) coupled with strong electromagnetic interaction across the flow. The range over which the controlling parameters in this study are varied has been arbitrarily chosen; however, these , values include conditions at which experiments have been made and therefore they are of practical interest.

Figure 2 is a plot of the azimuthal velocity distribution across the flow for the conditions denoted on the figure. This figure shows that the velocity is a rising function of the radius across the vortex and has the appearance of a solid-body rotation between the inner and outer electrode surfaces. The high value of the radial Reynolds number forces the viscous effects into a narrow region near the outer wall. As the ratio of the inner to the outer electrode radius is increased (increasing $\zeta_{1}$ ) the azimuthal velocity exhibits a marked decrease at all points in the flow. The distributions are similar to the lower $\zeta_{1}$ profiles, however, and the peak velocities occur at nearly the same location independent of $\zeta_{1}$. Results obtained with a radial Reynolds number of 10.0 indicate that the peak azimuthal velocities occur at a $\zeta$ value near 0.80 . For still lower values of the radial Reynolds number the viscous effects move farther out into the main part of the vortex, and the velocity distributions become similar to the zero radial mass flow profiles obtained by Kessey.

Figure 3 shows the pressure distributions that correspond to the previous results for the azimuthal velocity. All of the profiles shown in figure 3 are characteristic of solid-body rotation modified by the net mass flow through the chamber. Azimuthal injection of gas at the inner electrode surface does however raise the pressure within the vortex by as much as 27 percent above the zero azimuthal injection yelocity case for the conditions shown in figure 3. For $\mathrm{G}_{1}$ constant, the pressure at any point in the flow is strongly dependent on the boundary pressure $p_{0}$, as might be expected.

The temperature distributions corresponding to the previous conditions for the velocity and pressure are shown in figure 4 along with the particular choice of boundary temperatures. The distributions are nearly independent of the boundary temperatures that are chosen, and show an exponential behavior near the outer wall that is similar to the velocity distributions. Outside of the electrode boundary regions the joule heating terms are many times greater than the viscous dissipation and heat conduction terms and thus the assumptions made in arriving at equation (38) appear to be justified. The Mach number distributions across the flow were obtained from the previous results using the local speed of sound given in reference 9. Figure 5 shows the regions where sonic velocities are reached within the vortex. When $\zeta_{1}=0.7$, the entire vortex is subsonic as a result of the relatively short interaction length in the flow at this high value of $\zeta_{1}$. With the temperature and pressure known, the dependence of the electrical conductivity on zeta is known, and the power input can be obtained by numerical integration of equation (36). The power input divided by the chamber volume is shown as a function of the radius ratio $\zeta_{1}$ in figure 6 . The change in chamber volume with $\zeta_{1}$ produces large differences in the total power required. For the conditions shown in this figure, the total power required when $\zeta_{1}=0.3$ is nearly four times the power required for the $\zeta_{I}=0.7$ case. Figure 7 shows the induced azimuthal magnetic field distribution for the case where $\zeta_{1}=0.3$. In this figure $B_{\theta}$ is plotted as a function of the axial coordinate $\xi$. The cylindrical surfaces of the vortex chamber determine the maximum and minimum values of the flux distribution shown in the figure. For symmetrical current flow through each end of the center electrode the induced magnetic field has the direction shown in figure 7 for each half of the chamber. The flux distribution is symmetrical about the z-axis and the curves plotted in figure 7 show that the largest axial forces on the plasma will occur near the end walls of a finite length chamber. These forces are in a direction that tends to move the plasma away from the end walls toward the axial midplane of the chamber when positive current flows in the outward radial direction.

The dependence of the azimuthal velocity on the Hartmann number is not contained explicitly in equation (24); however, the effect of different values of this number can be indicated by plotting

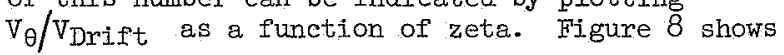
this velocity ratio for the conditions indicated. Similarly, figure 9 shows the pressure ratio, $\frac{p}{\frac{1}{2} p^{2} \text { Drift }}$ as a function of zeta. In both the previous figures the drift velocity is evaluated at its maximum value near the outer wall. Higher values of the Hartmann number can be viewed as yielding higher azimuthal velocities although figure 9 shows a significant flattening of the pressure ratio as the Hartmann number is decreased. From a practical standpoint, the shallower pressure profiles are desirable because they tend to reduce secondary flows whthin the end-well boundary layers.

With the radial and azimuthal velocity known at each point within the vortex, fluid streamlines can be plotted and figure 10 shows a typical fluid element streamline for the conditions denoted. For low values of $\zeta_{1}$ and high values of the electromagnetic parameter $\mathrm{Ni}$, it is apparent that large interaction lengths can be obtained within moderately sized chambers. 


\section{Experimental Measurements}

Experiments have been made with several arcdriven vortex accelerators, and diagrams of these devices are shown in figures $11(a)$ and $11(b)$. Farly experiments with the device shown in figure 17 (a) are reported in reference 5 , and subsequent modifications to both accelerators have provided a means for gas injection with subsonic and supersonic azimuthal velocities on each side of the center electrode. The radial injection velocity is subsonic for all of the experiments reported here. Dral light pipe viewing stations in the accelerator exit channel have allowed direct measurements of the exit gas velocity to be made and compared with velocities obtained from simultaneous solutions to the energy and mass continuity equations and the equation of state using measured quantities as inputs to these equations. A gas accelerating chamber with an axial length of 0.635 centimeter was used with the end walls recessed as indicated in figure 11(a). Also, experiments with a 3.8-centimeter-long chamber and a 2.I-weber/meter ${ }^{2}$ applied magnetic field were carried out with the device shown in figure $11(\mathrm{~b})$. A comparison between measured quantities and values obtained from the theoretical flow model is subject to a number of limitations. The experimental devices have a single azimuthal gas exit rather than the porous wall assumed in the flow model. Inlet gas is injected on each side of the center electrode and not through the electrode surface. Also, there is a periodic motion of the conducting gas within the chamber of the experimental devices which is analogous to a rapidy rotating arc rather than an axisymmetric plasma. (Arc-rotational frequencies up to 28,000 revolutions per second have been measured within the chamber for some of the tests reported in this study.) An experimental indication of the validity of the assumption that the flow is axisymmetric has been obtained with a rapid response pressure transducer (piezoelectric crystal) mounted within the outer wall. For several different chamber pressure levels, the magnitude of the periodic pressure fluctuations was a small fraction of the time-averaged outer wall pressure. In addition, time-averaged pressures measured on the inner electrode surface and around the outer wall showed almost no deviation from an axisymetric distribution existed. On the other hand, nonaxisymmetric heating from the arc was not investigated. In the experiments, use of time-averaged energy input to the gas may lessen the validity of a comparison with an axisymmetric model. Because of the long interaction length within the chamber, however, the effects of nomuniform heating tend to be reduced. Finally, all of the input parameters to the theoretical results cannot be uniquely specified from the experimental measurements. In particular, the experimental chamber is of finite length which has the effect of altering the radial mass flow through the vortex. Also, the radial current is measured rather than the radial current density. With these considerations in mind, figure 12 shows three theoretical velocity distributions for the range of conditions of the experiments. Also shown are three experimental values of velocity in the outer flow. The agreement between the theoretical and measured velocities at $\zeta=0.875$ is quite reasonable.

For $\zeta_{1}=0.6$, figure 13 shows both the theoretical and measured pressures at the vortex boundaries that correspond to the previously discussed velocity results. The calculated and measured values of applied voltage are shown in figure 14 . If anode and cathode fall voltages were subtracted from the measured voltage, some disagreement would exist between the calculated and the experimental values of applied potential. These results may well be due to an effectively higher electrical conductivity in the arc than is obtained from the theoretical model. Finally, the calculated and measured power input to the vortex is shown in figure 15 . For $\zeta_{1}=0.5$, the experimental power input is

11 percent above the calculated result. The neglect of electrode boundary losses in this analysis may be primarily responsible for this discrepancy. For $\zeta_{1}=0.6$, the measured power input is 37 percent below the calculated value. An explanation for the result with $\zeta_{1}=0.6$ is difficult to justify and further experimentation must be made to resolve this question.

One point of interest regarding the experimental observations concerns the induced azimuthal magnetic field. The calculated values of $B_{\theta}$ indicate that it is a small quantity at all locations within the vortex chamber. Early tests, however, were made with the total current introduced through one end of the center electrode and, as might be anticipated, a net axial Lorentz force drove the plasma against one end wall and caused an axial component of current to flow. By introducing an equal value of current through each end of the center electrode the axial forces on the plasma cancel each other and appear to result in a purely radial current flow.

Experiments with the device shown in figure $11(b)$ have shown large increases in azimuthal velocity and energy input to the gas in comparison with the previous measurements. With an applied magnetic field strength of 2.1 webers/meter ${ }^{2}$, a current of 1300 amperes, and a radial Reynolds number of 330 , the measured total gas enthalpy is $38 \times 10^{6}$ joules $/ \mathrm{kg}$ which is 1.8 times the enthalpy obtained with the device shown in figure Il(a). Pressure measurements made on the outer wall show an axisymmetric distribution and figure 16 shows a measured velocity and the predicted velocity distribution. The applied voltage is shown in figure 17 and the power input is shown in figure 18 . Further experiments will allow at least a gross comparison of the differences between the devices shown in figure 11 (a) and $11(b)$. The device shown in figure $11(b)$ is exhausted to low pressure through a $10^{\circ}$ diverging nozzle that has a 24 centimeter by 24 centimeter square exit. Velocity and pressure measurements made in the nozzle and at the exit indicate a nearly isentropic expansion that produces a 15-centimeter by 15-centimeter core at the exit. Lateral and vertical pitot probe traverses show a symmetrical pressure distribution about the axis. The nozzle exit static pressure is 0.45 millimeter of mercury, the stream velocity is 6400 meters/second, and the Mach number is 5.1 when pure nitrogen is used as the test gas. As yet no limit to the maximum mass flow that can be heated and accelerated has been established, although higher power input is required as the mass flow and chamber pressure are increased. Experiments at considerably higher power levels than are reported here have been initiated.

Considering the highly idealized flow model chosen for this study, the agreement between measured and predicted flow behavior seems encouraging. 
If, as it appears, solid-body-like rotation exists within the experimental devices, then favorable conditions exist for producing considerably higher gas velocities and gas energies than have been obtained up to the present time.

\section{Concluding Remerks}

1. The basic nature of the electromagnetically driven vortex studied in this analysis is governed by the coupling between the azimuthal Lorentz force and the high positive radial Reynolds numbers that are brought about by outward radial mass flow.

2. All of the calculated results exhibit a strong dependence on the inner to outer electrode radius ratio. For small values of this ratio the solid-body-Iike azimuthal velocity distributions produce high gas velocities in the outer portions of the vortex.

3. At currents near 1500 amperes and magnetic field strengths of 1.0 and 2.1 weber/meter ${ }^{2}$, reasonable agreement exists between the theoretical and experimental results regarding the velocities in the outer portion of the vortex, the inner and outer boundary pressures, and the power required to heat and accelerate the flow.

4. For chamber pressure levels of 1 atmosphere and greater, high energy input to the working fluid appears possible by the heating and electromagnetic acceleration considered here. In addition, high peak velocities are attainable within practical electrode and magnetic field geometries.

\section{References}

1. McCune, J. E.: Theory of Magnetohydrodynamic Homopolar Generator. Part II, Gaseous Mediums, Aeron. Res. Asso. of Princeton, ARAP Rept. No. 25, Princeton, N.J., 1960.

2. Donaldson, C. D.: The Magnetohydrodynamic Vortex Power Generator Basic Principles and Practical Problems, Aeronautical Res. Asso. of Princeton, ARAP Rept. No. 30, Princeton, N.J., 1961.

3. Lewellen, w. S.: Magnetohydrodynamically Driven Vortices, Proc. of 1960 Heat Trans. and Fld. Mech. Inst., Stanford Univ., 1960.

4. Kessey, K. 0.: Rotating Electrically Conducting Fluids in a Long Cylinder, AIAA Journal, Vol. 2, No. $5,1964$.

5. Stewart, R. B.; and Wallio, M. A.: The Experimental Performance of an Are Driven Vortex Type Heater Accelerator. Presented in Fourth Hypervelocity Tech. Symp., Tullahoma, Tenn., 1965.

6. Sutton, G. W.; and Shermen, A.: Engineering Magnetohydrodynamics. McGraw Hill Book Co., 1965.

7. Kessey, K. O.: Magnetohydrodynamic Rotation of Plasmas. Columbia Univ. Plasma Lab., Rept. 1, 1963.

8. Viegas, J. R.; and Peng, T. C.: Flectrical Conductivity of Ionized Air in Thermodynamic Equilibrium, American Rocket Soc. Jour. 31, $654,1961$.

9. Hansen, C. F.: Approximations for the Thermodynamic and Transport Properties of High Temperature Air. NASA TR R-50, 1959. 


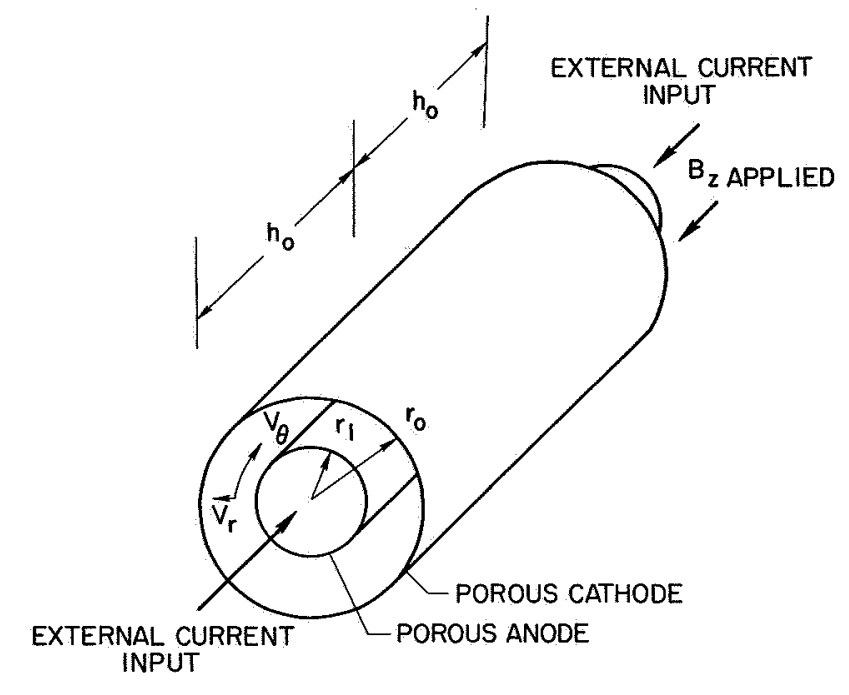

Figure 1.- Electromagnetically driven vortex geometry.

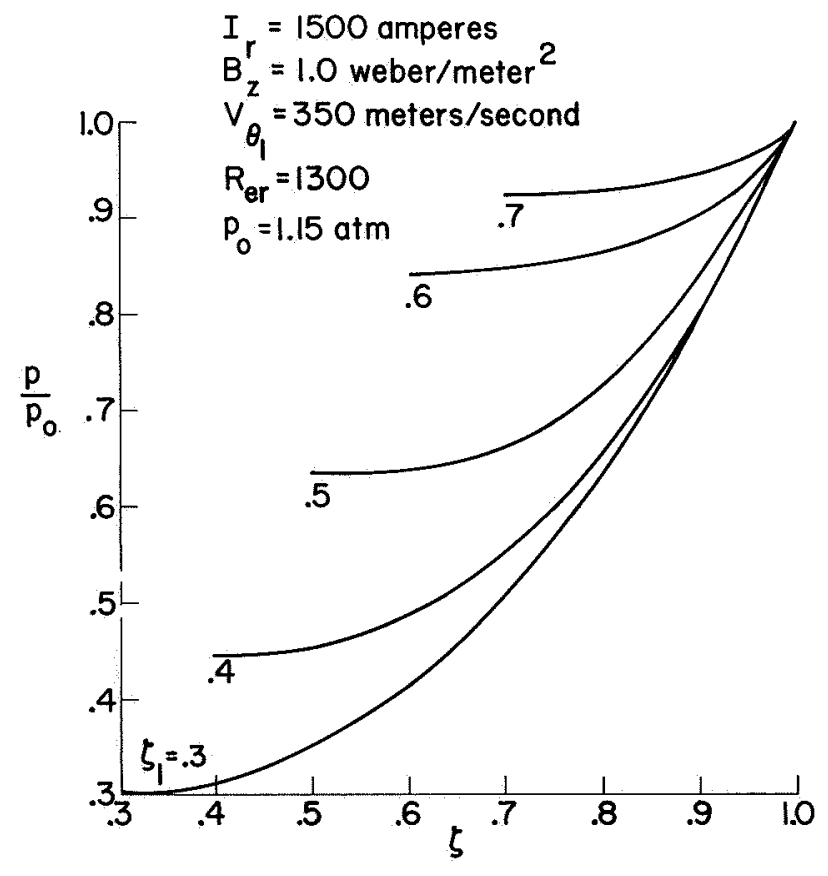

Figure 3.- Pressure distribution across vortex.

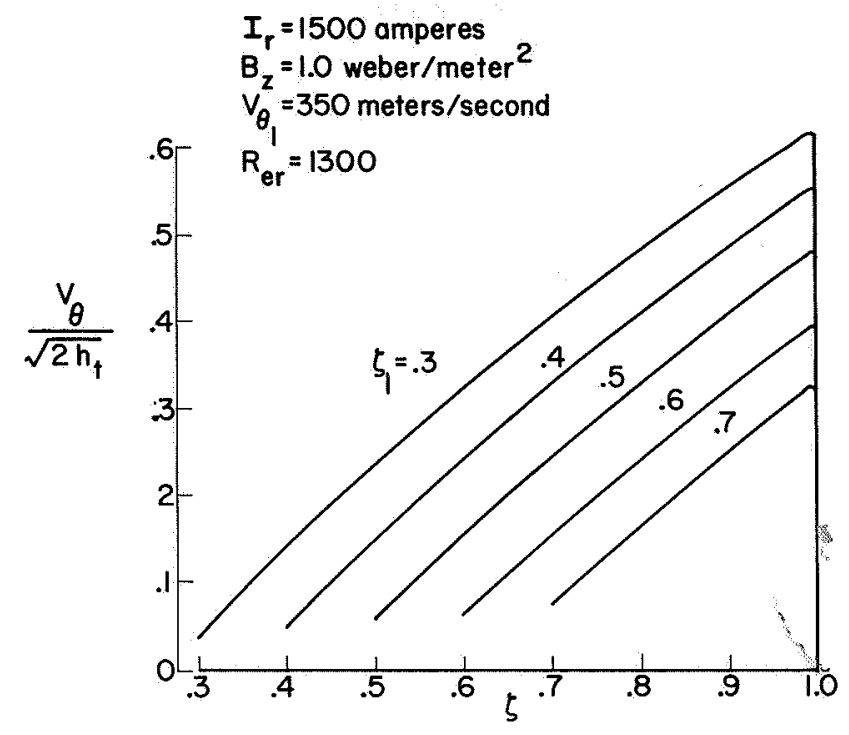

Figure 2.- Azimuthal velocity distribution across vortex.

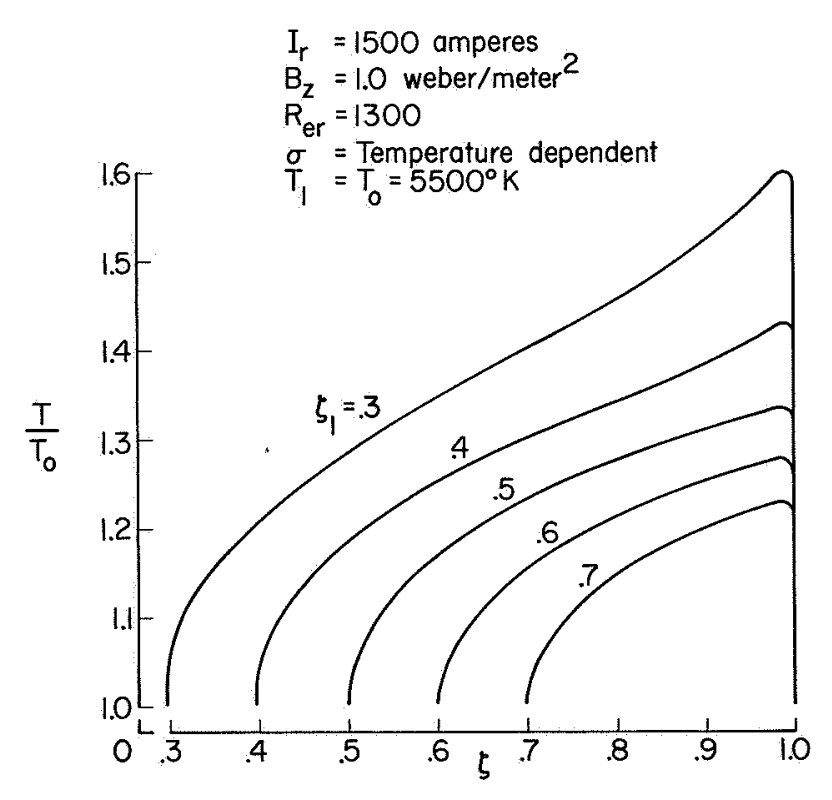

Figure 4.- Temperature distribution across vortex. 


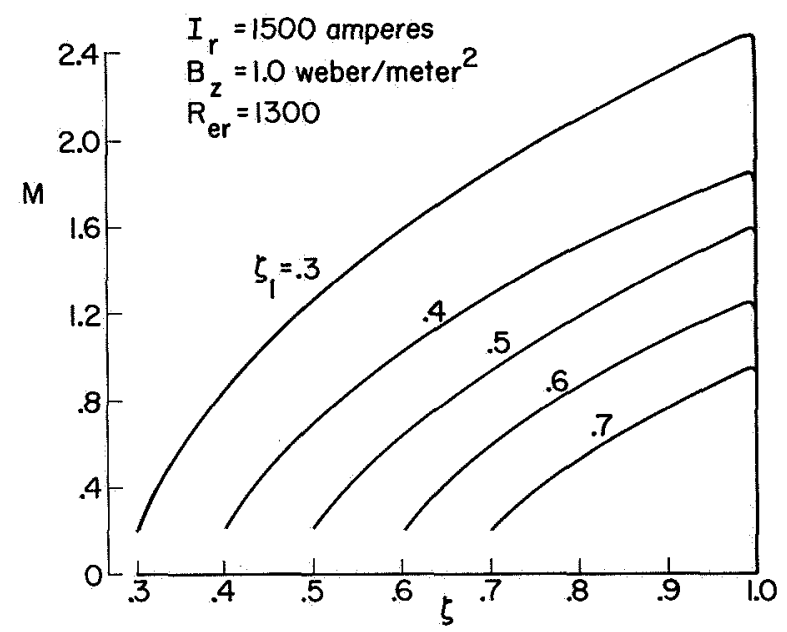

Figure 5.- Mach number distribution across vortex.

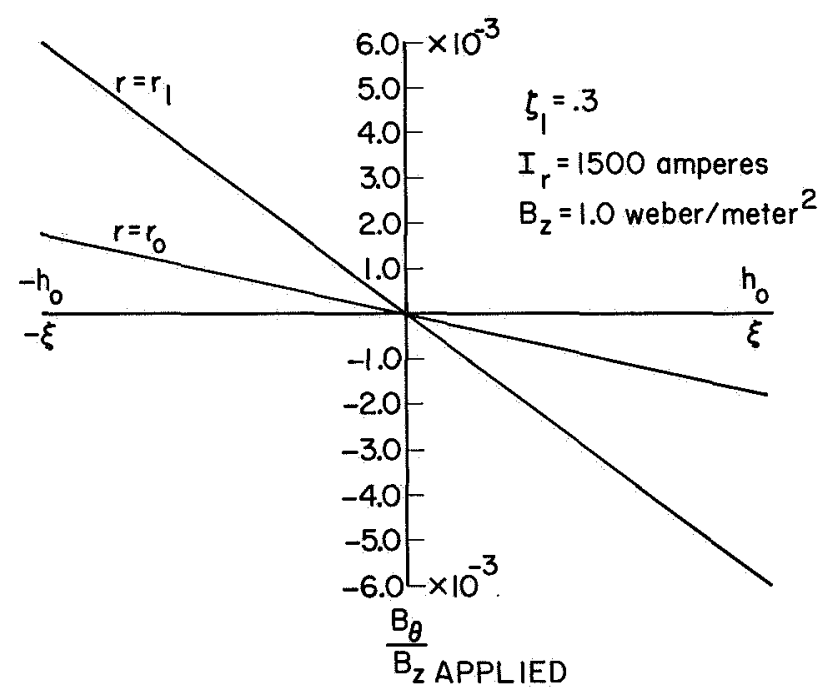

Figure 7.- Induced azimuthal magnetic field distribution.

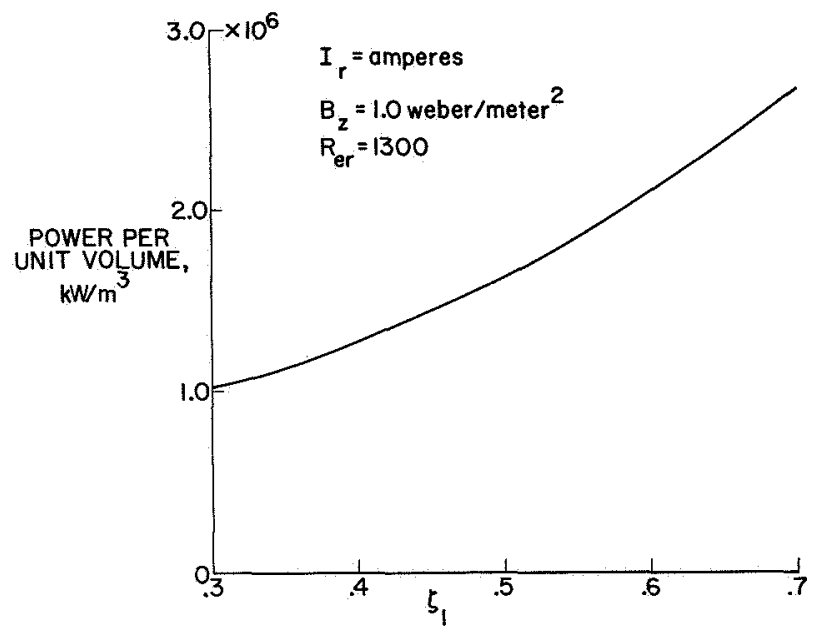

Figure 6.- Power input variation with radius ratio.

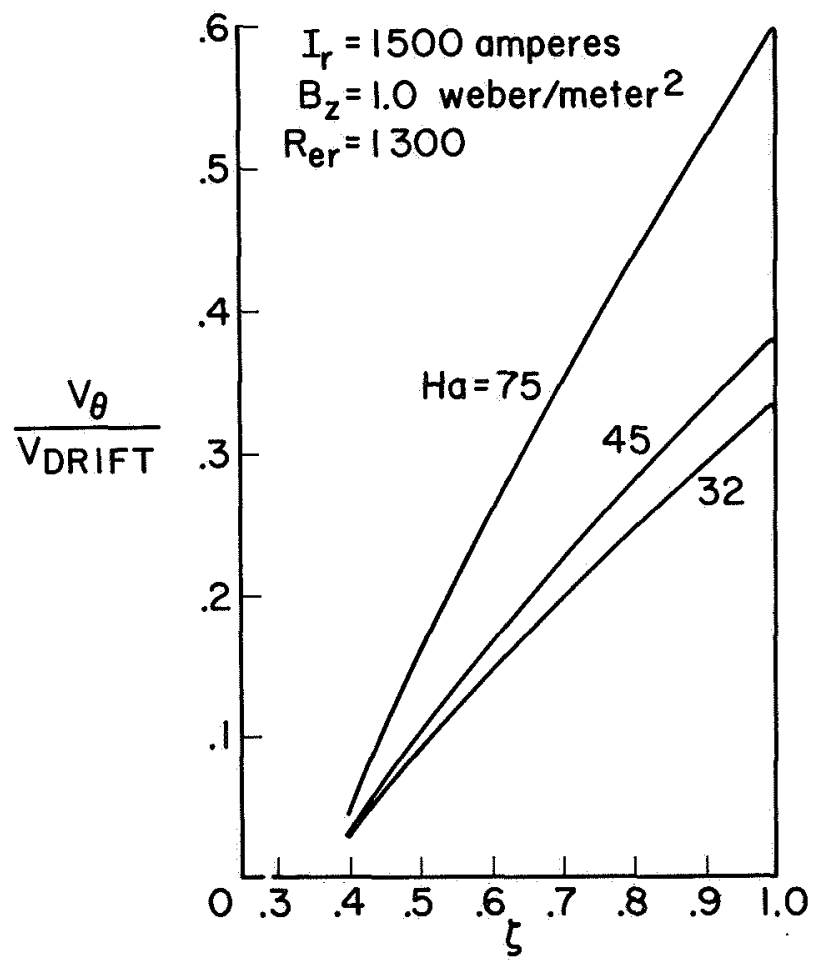

Figure 8.- Hartmann number effect on azimuthal velocity. 

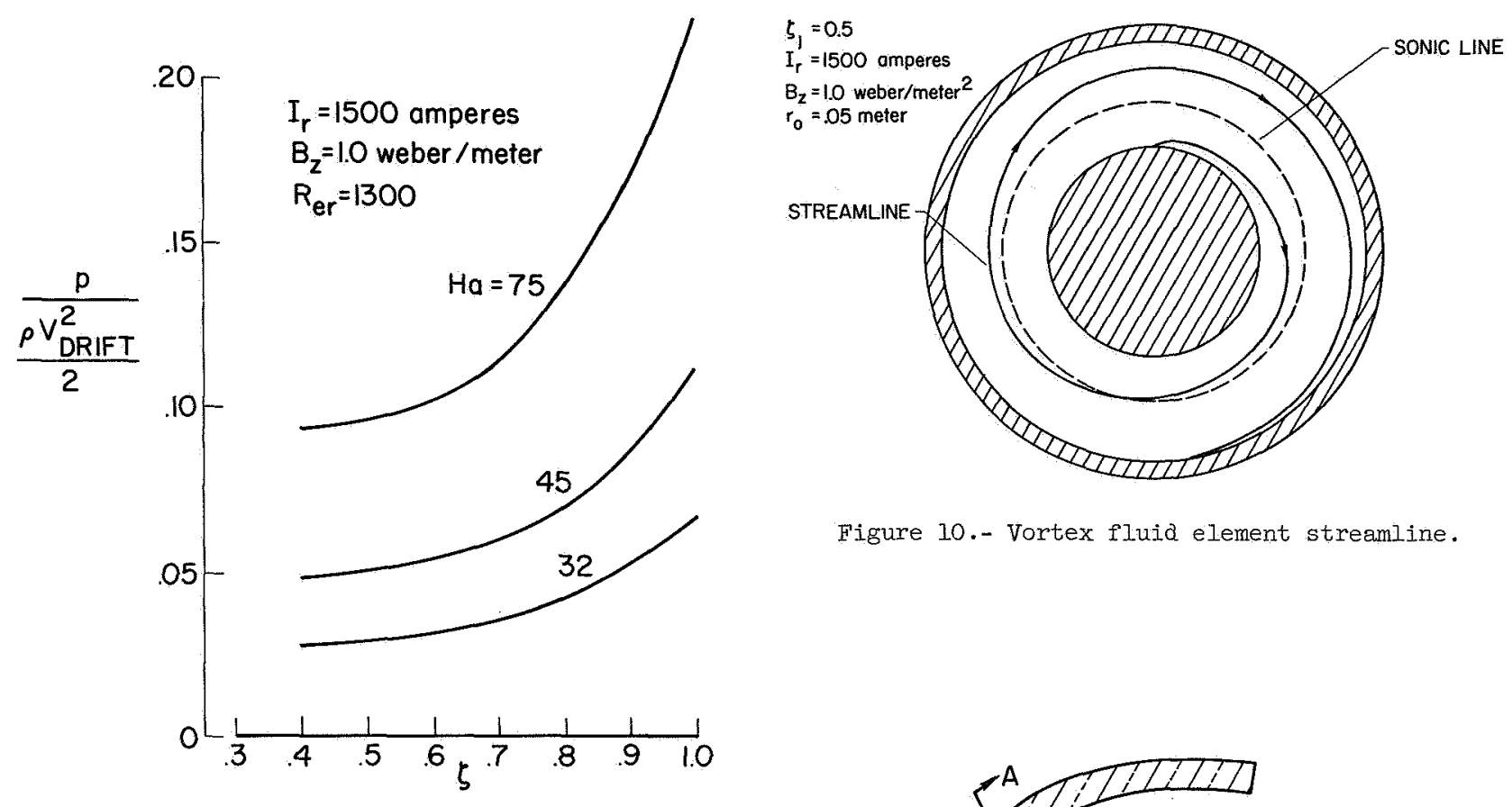

Figure 10.- Vortex fluid element streamline.

Figure 9.- Hartmann number effect on pressure distribution.
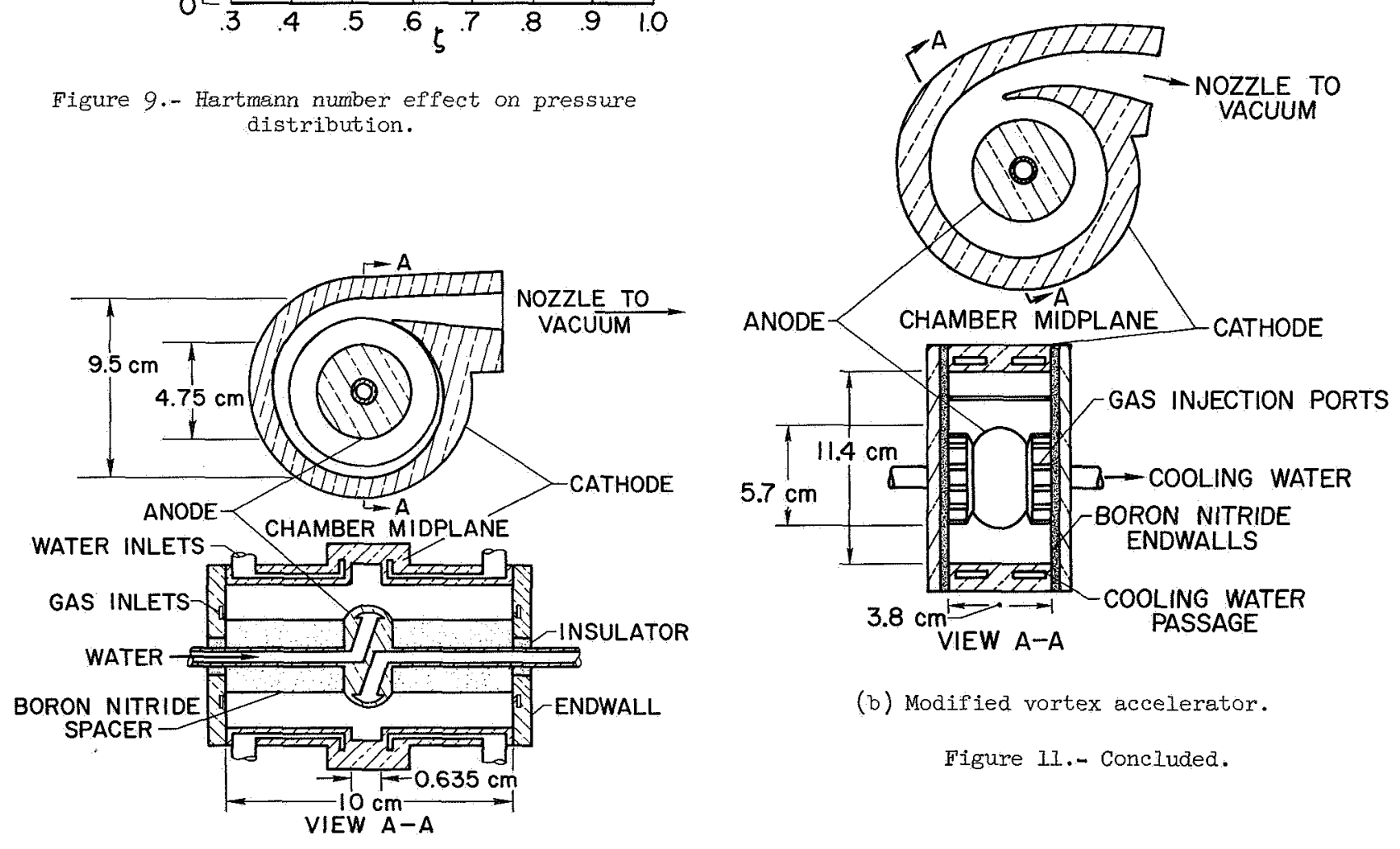

(b) Modified vortex accelerator.

Figure 11.- Concluded.

(a) Vortex accelerator geometry.

Figure 11. 


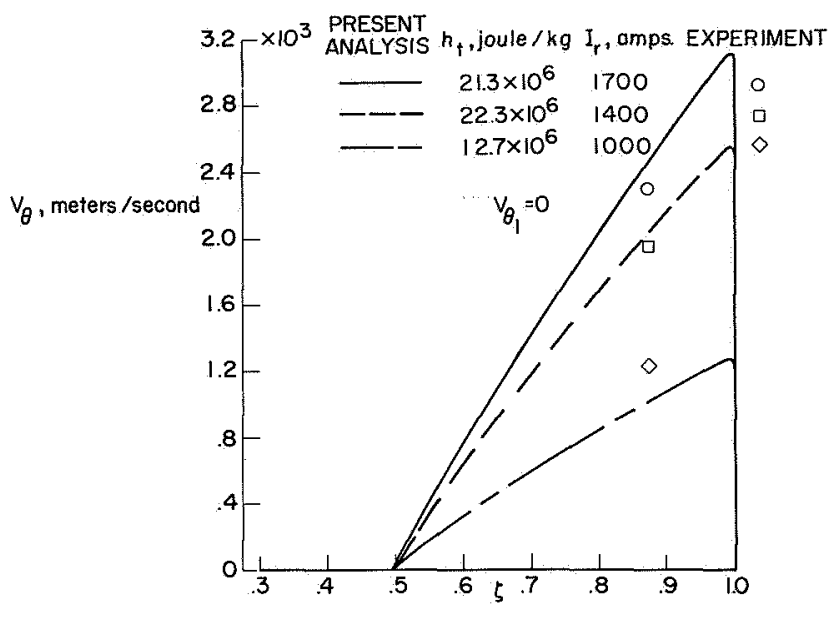

Figure 12.- Experimental azimuthal velocities.

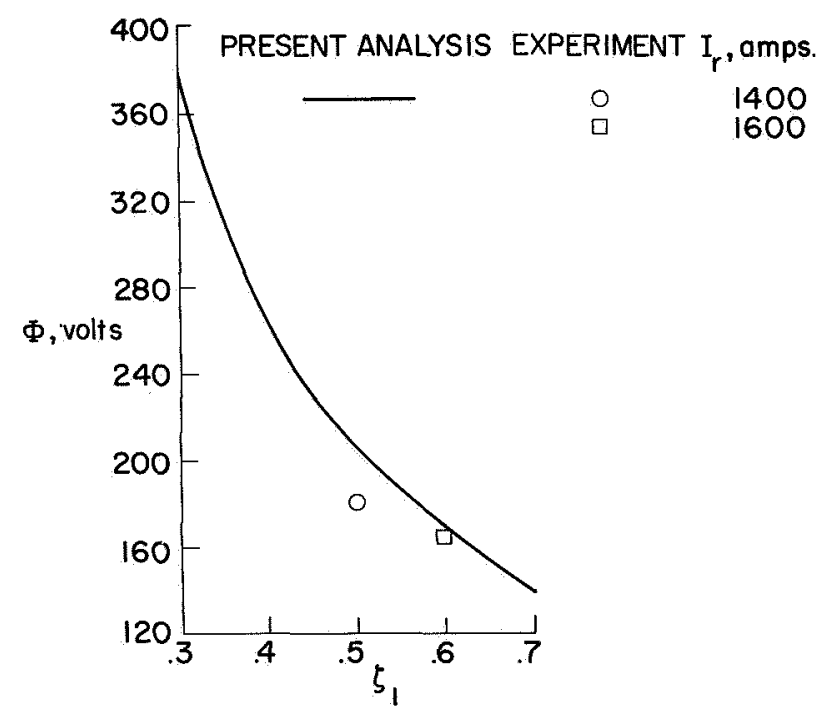

Figure 14.- Experimental applied voltage.

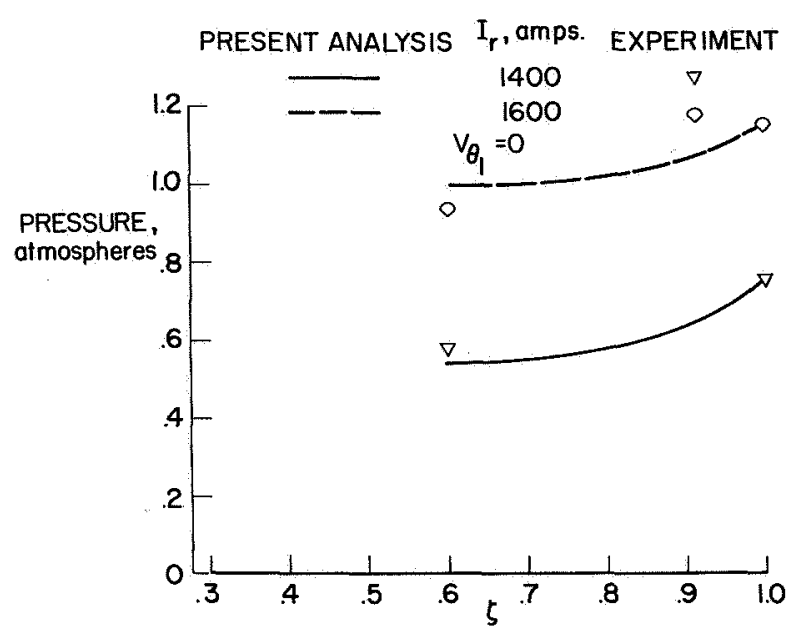

Figure 13.- Experimental pressure at vortex boundaries.

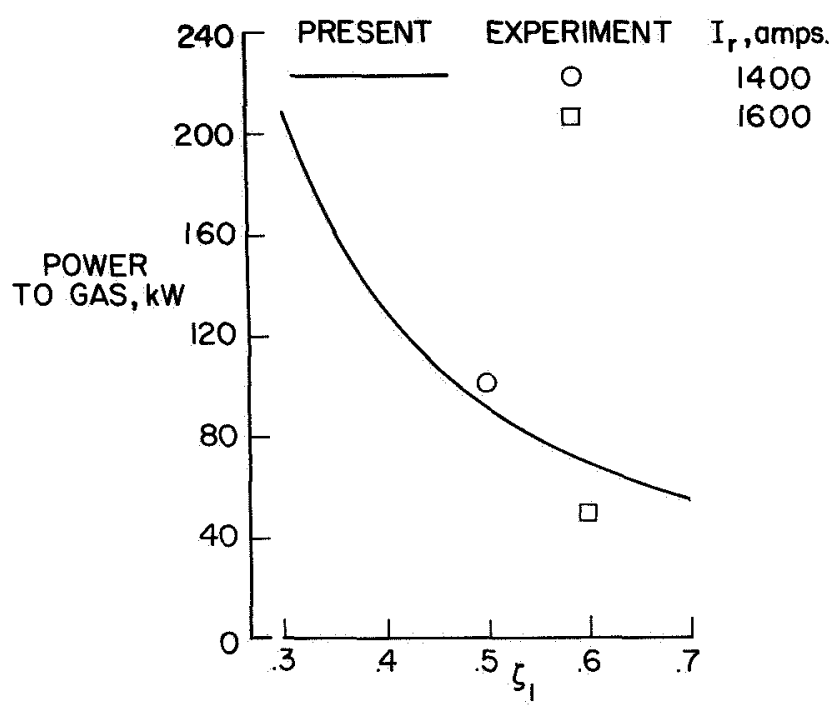

Figure 15.- Experimental power input to vortex. 


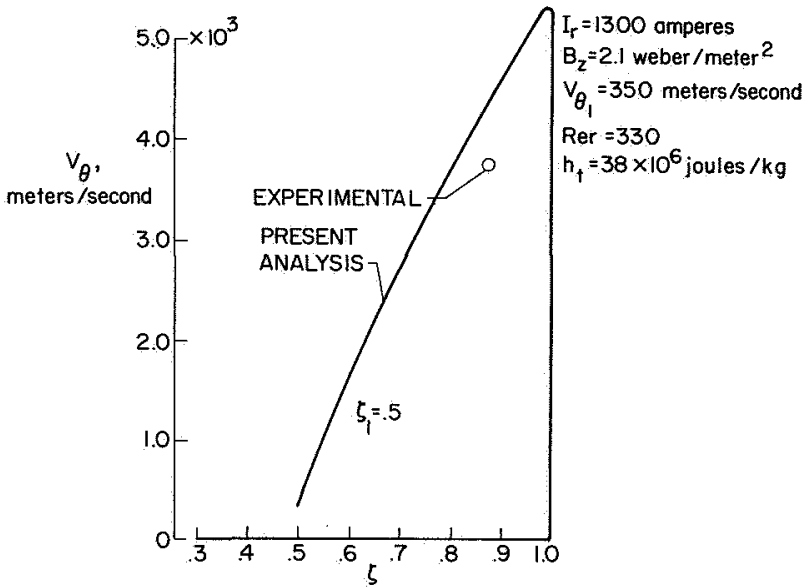

Figure 16.- Experimental azimuthal velocitymodified vortex accelerator.

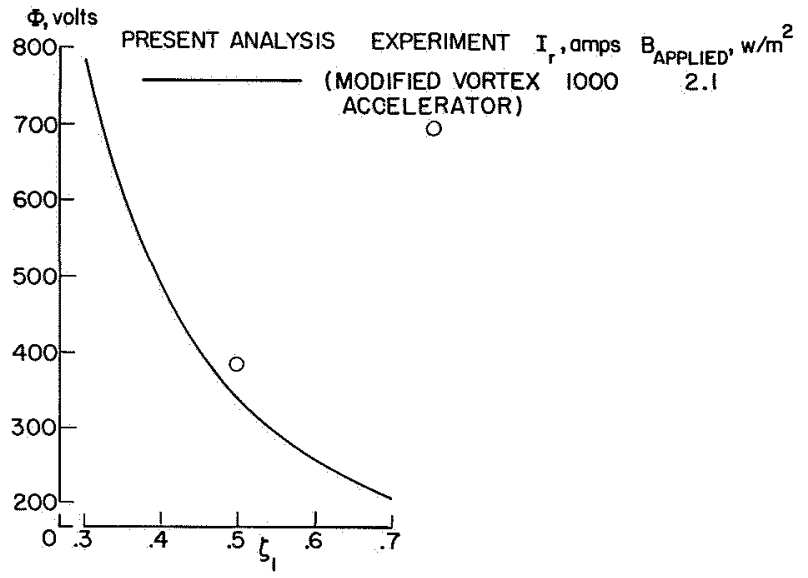

Figure 17.- Experimental applied voltage.

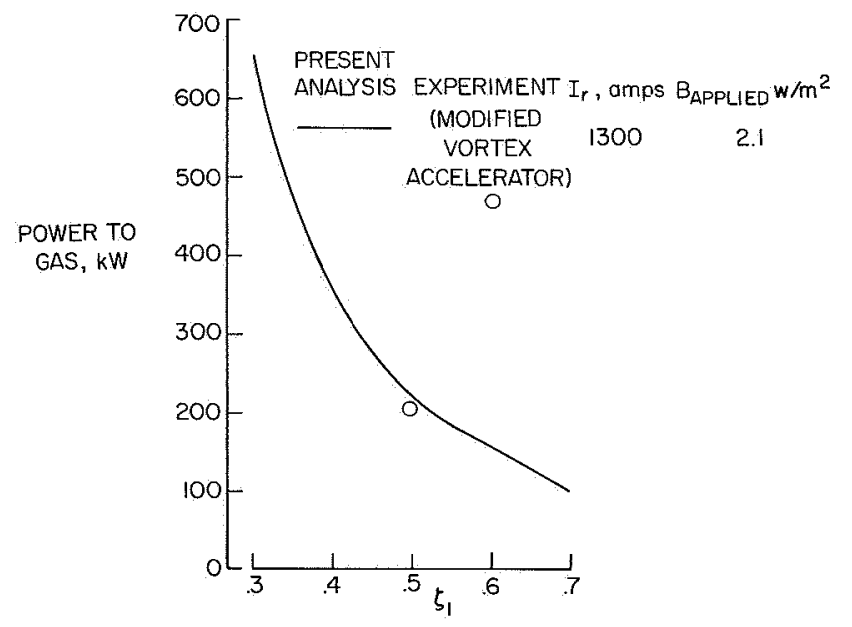

Figure 18.- Experimental input power to vortex. 